\title{
Modified Heyman packing performed with small number of Simon-Norman catheters
}

\author{
Jenö Pálvölgyi, MSc, PhD, Kofi Agyemang-Prempeh, MD \\ Department for Oncoradiology, Petz County Teaching Hospital, Györ, Hungary
}

\begin{abstract}
Purpose: Modified Heyman packing method, performed usually with 6-11 flexible catheters is one out of many brachytherapy treatments. We described the insertion and dose planning method, with the use of 4-6 Simon-Norman catheters positioned to the fundus uteri, which is the most frequent source of the endometrial cancer.

Material and methods: We investigated the dose distribution of the main classes of regular catheter insertions with Simon-Norman applicators using dose profiles, obtained in lateral and axial direction at the fundus, and compared with the dose distribution of the Y-shaped catheter arrangements.

Results: The insertions with five and six Simon-Norman catheters produced higher dose at the fundus uteri, compared with the Y-shaped applicator.

Conclusions: Insertions performed with small number of catheters permits to perform dosimetry optimisation for each treatment fraction.
\end{abstract}

J Contemp Brachyther 2010; 2, 1: 33-36

Key words: endometrial carcinoma, Heyman packing, Simon-Norman catheter.

\section{Purpose}

Surgery with clinical staging to determine the need for additional adjuvant therapy is a recently accepted protocol for treatment of endometrial cancer [1]. However, medically inoperable patients can be treated by brachytherapy (BT). The BT irradiation can be performed with standard rigid applicators such as tandem or $\mathrm{Y}$ shaped applicators with three or four channel applicators [2], or by modified Heyman packing method [3]. The most important prognostic factors for carcinoma of corpus uteri treatment are: the staging, dimensions of the organ, the depth of uterine cavity and the thickness of myometrium. Dimensions of the uteri can be obtained by magnetic resonance scans (MR) [4] or by ultrasonography (US) [5].

Brachytherapy insertions using the modified Heyman packing method according to Herbolsheiner et al. are performed with 6-11 pieces of Simon-Norman catheters. The heads of the catheters are uniformly distributed in the uterine cavity $[3,6]$. Reconstruction of the large numbers of catheters is a time consuming process. Erikson et al. reduced the treatment planning time with fractions performed with standard plans [7]. Our primary aim was to develop an insertion technique for treatment of the fundus uteri which is the most frequent origin of endometrial cancer [8] and we intended to prepare individual treatment plan for all treatment fractions.

\section{Material and methods}

Endometrial insertions with smaller amount of SimonNorman catheters (Nucletron ${ }^{\circledast}$ ) was performed. Depending on the dimension of the uterus, the number of catheters were 4-6. Another important factor was the heads of all the Simon-Norman catheters positioned to the fundus uteri, as illustrated in Fig. 1. We inserted five or six catheters with $6 \mathrm{~mm}$ diameter into the medium and the large uterus, respectively. In case of the small size of the uterus, the number of catheters were 4 to 5 with two or three catheters positioned towards the fundus. The catheter insertion was performed in lithotomic position. After finishing the procedure, pairs of posterior-anterior (PA) and posterioroblique (PO) reconstruction images with the C-arm located in brachytherapy treatment room was obtained. The video signal from the $\mathrm{C}$-arm monitor was frame grabbed and the images were converted to Dicom ${ }^{\circledast}$ format [9]. The catheter reconstruction and dose calculation was performed by Plato Brachytherapy Treatment System v13.7 (BPS) (Nucletron ${ }^{\circledast}$, Netherlands). In order to obtain dose distribution, which follows the shape of the uterus, the reconstruction of the laterally outermost left and right catheters with full length in the uterine cavity was performed, and the other - with limited length of clear separation of the catheters. We generated dose points in a lateral distance of the uterine wall thickness from the outermost left and right catheters, as illustrated in Fig. 2.

\footnotetext{
Address for correspondence: Jenö Pálvölgyi, MSc, PhD, Department for Oncoradiology, Petz County Received: 27.01 .10 Teaching Hospital, H-9002 Gyor, Pf 92. Zrínyi str 13, Hungary, phone +36-96-418-244, fax +36-96-433-671, Accepted: 07.03.10 e-mail: palvolgyij@petz.gyor.hu Published: 28.03 .10
} 


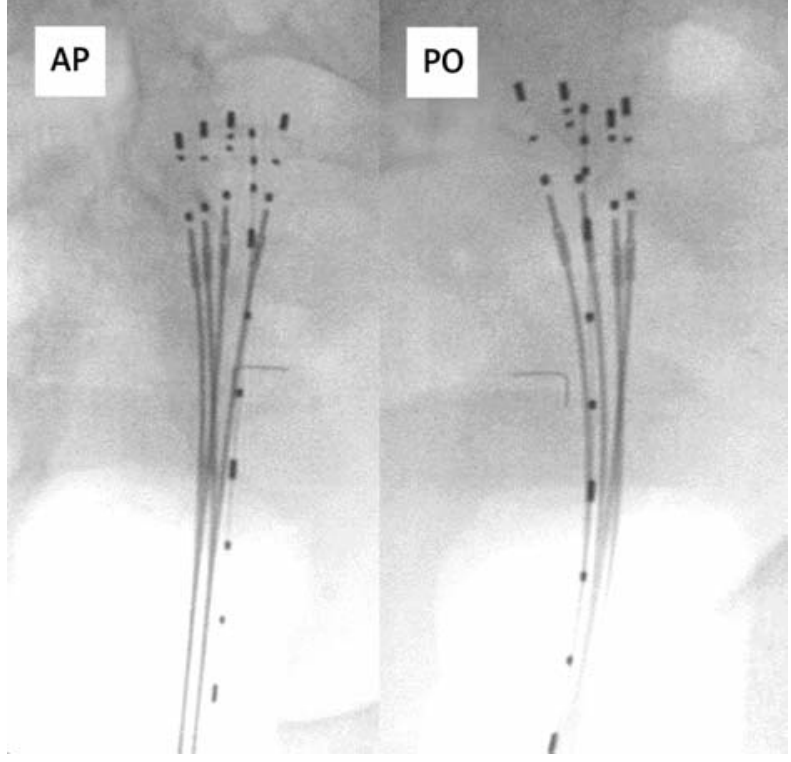

Fig. 1. Anterior-posterior $(\mathrm{AP})$ and posterior oblique $(\mathrm{PO})$ fluoroscopy images of insertion performed with five SimonNorman catheters with $6 \mathrm{~mm}$ diameter head. Each catheters head is positioned to the fundus uteri

The uterine wall thickness was measured on the basis of MR images or ultrasound performed prior to BT treatment. The dose distribution was optimised with dwell time settings, using optimisation method "on dose points and geometry" implemented in Plato BPS ${ }^{\circledR}$.

Location of the Simon-Norman catheters is different in each insertion and in each treatment fraction. We experienced types in geometrical arrangements of Simon-Norman catheters, illustrated in Fig. 3. Dose distribution of the most frequent types of idealized catheter arrangements was assessed. We modelled the catheter location in the uterus, fixing the Simon-Norman catheters together and imitating regular catheter arrangements. We obtained pairs of reconstruction images and prepared treatment planning. We assessed the dose distribution of models insertion, converting 3D dose-matrices, generated by BPS with $3 \mathrm{~mm}$ resolution to Dicom3 format resulting in multi-slice images of 100 slices: each $100 \times 100$ pixels. The brightness of the pixels represents the dose value like the autoradiography image. The appropriate slice and generated axial and lateral dose profiles with the Image J $1.42 q$ program (http://rsb.info.nih.gov/ij) was selected. We prepared two dose plan modifications: one with all catheters activation, and the second - with two laterally outermost left and right catheters activation. We determined the origin of dose profiles with the later plan variant by the midpoint of the first source position of the outermost left and right catheters.

\section{Results}

Figure 3 presents the AP views of three classes of catheter arrangements, the axial and lateral dose profiles for each treatment plan modifications. The lateral dose profiles showed small differences, while dose profiles obtained in axial direction from catheters heads demonstrated that five and six catheters arrangements provides at least two times higher dose in the uterine wall at the fundus, compared to dose distribution of two channels plan option. Small difference was found in axial dose profiles in two under three ( $3+2$ in Fig. 3$)$ arrangement, either activating all catheters $(n=3+2)$ or activating three catheters at the fundus only $(n=3)$.

\section{Discussion and Conclusions}

Reconstruction of Simon-Norman catheters are based on pairs of biplane images in addition to sectional BT guided images [6]. At our institutions we introduced the use of
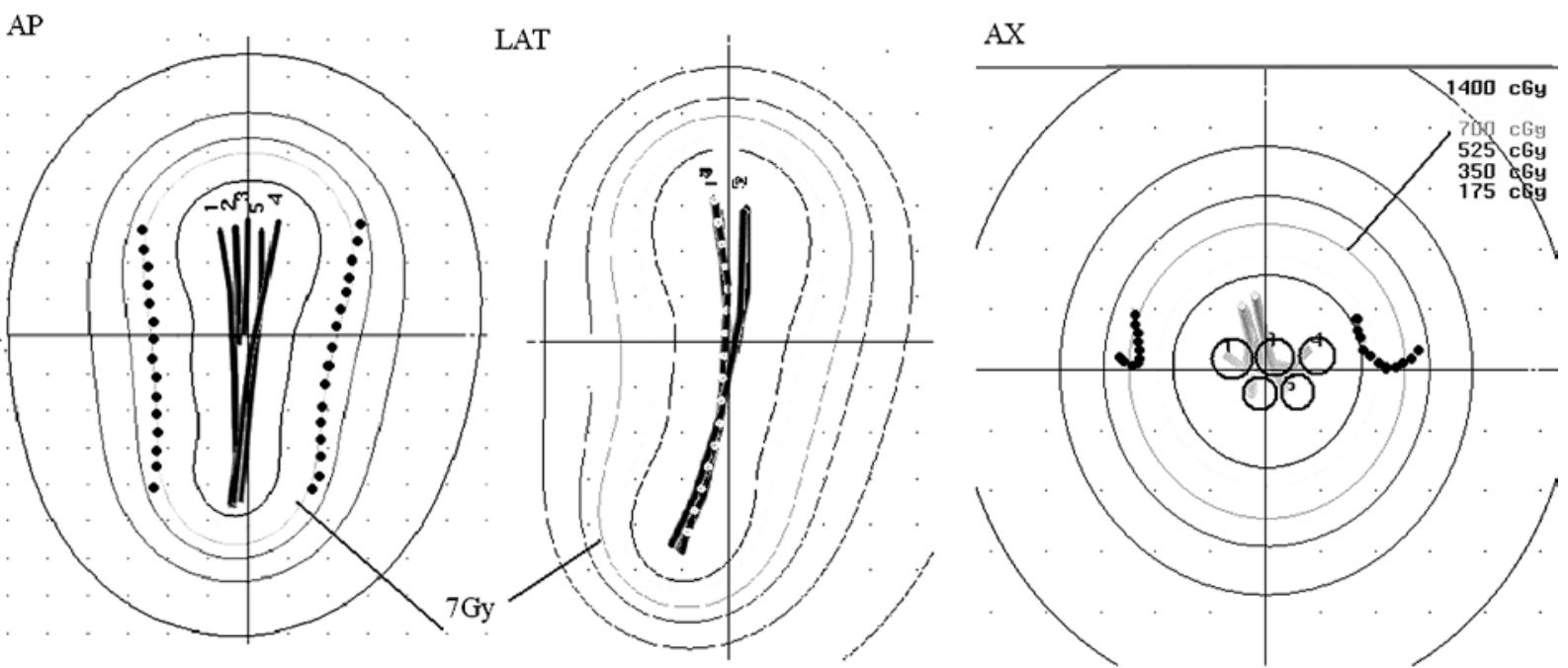

Fig. 2. Anterior-posterior (AP) and lateral (LAT) views of reconstructed catheters and the isodose curves normalized and optimized for dose points defined in a distance of the uterine wall thickness from the laterally outermost left and right catheters (\#1 and \#4). These catheters were reconstructed in full length in the uterine cavity, while the others were reconstructed in close proximity to the fundus. The axial view (AX) of the reconstructed catheters at the fundus, the circles represent the heads of the Simon-Norman applicators 

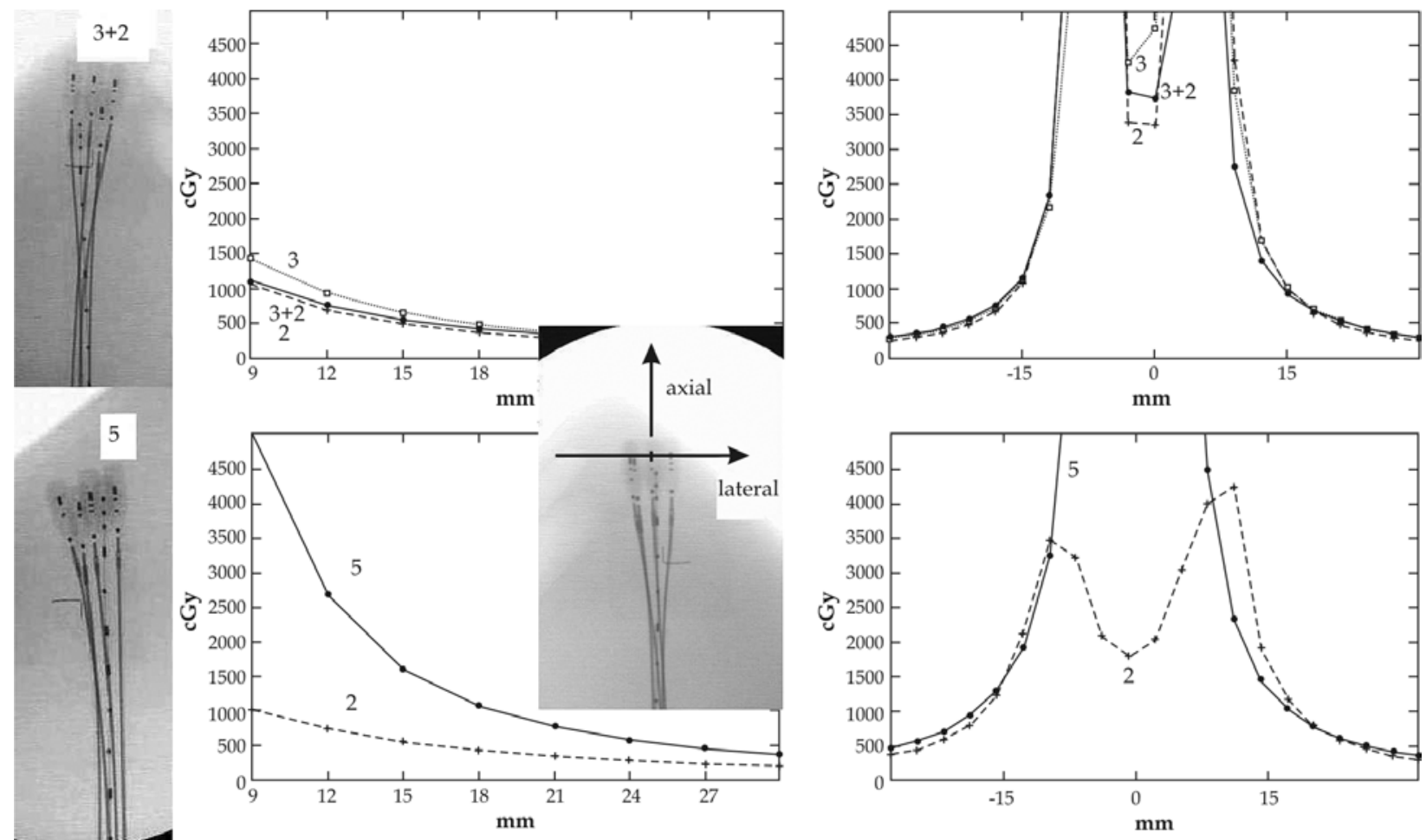

6
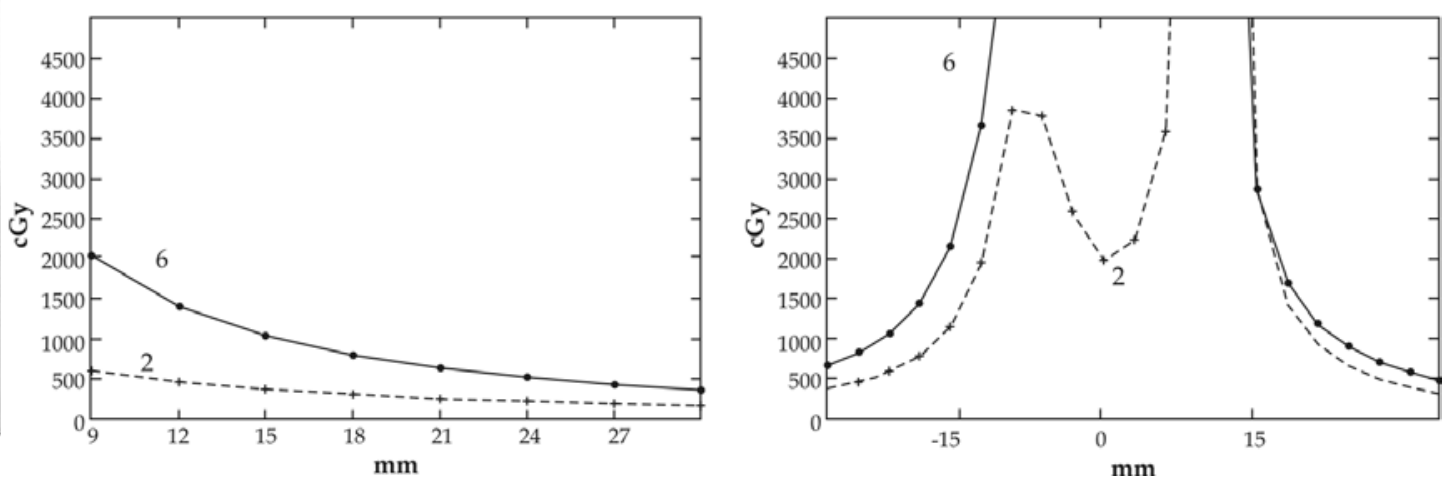

Fig. 3. Fluoroscopy images of main types of Simon-Norman catheter insertion models: two under three $(3+2)$, five and six catheters arrangements. The corresponding axial dose profiles from catheters heads and the lateral dose profiles obtained from the multichannel and two channels dose planning (2)

pairs of PA and PO images obtained with angle between 135-150 degrees. We use PO images in gynaecological applicator reconstruction instead of typical lateral applicators. In PO image the shielding of bony structures is smaller than in lateral, resulting better quality of images [9]. We selected angle of the PO image with no overlapped catheters. The insertion technique performed with small number of Simon-Norman catheters for treatment of carcinoma endometrium has advantages as follows: in PA images the catheters located closely to the fundus are separated and the identification codes of radio-opaque markers can be visibly distinguished. We experienced good match in uterine dimensions measurements on the basis of MR images obtained prior to BT treatment and uterine dimension calculations acquired by the co-ordinates of reconstructed catheters [10]. This indicates that the insertion of small amount of Simon-Norman catheters do not significantly change the uterine dimension. The flexible catheters follow the shape of the uterus. The dose optimization with dose points defined in a distance of the uterine wall thickness from the laterally outermost left and right catheters provide acceptable dose coverage especially in case of medium size uterus [10]. The dose distribution of the rigid Y-shaped applicator provide high dose to the uterine corners, while five and six channels insertions present considerably higher dose in the uterine fundus compared with two channels applicator. The catheter arrangements in multiple treatments are relatively different. Insertions performed with small number of catheters permit to perform dosimetry optimisation for each treatment fraction. 


\section{References}

1. Practice Guidelines: Uterine Corpus - Endometrial Cancer. Oncology 1998; 12: 134-138.

2. Bauer M, von Fournier D, Fehrentz F et al. Afterloading method for simulating the intrauterine packing method in case of carcinoma of the body of the uterus. Strahlentherapie 1981; 157: 793-800.

3. Herbolsheiner M, Sauer O. HDR Brachytherapy for Endometrial Cancer. International Nucletron Radiotherapy Journal 1996; Special Report No.8: 34-45.

4. Lee EJ, Byun JY, Kim B et al. Staging of Early Endometrial Carcinoma: Assessment with T2-weighted and Gadoliniumenhanced T1-weighted MR Imaging. Radiographics 1999; 19; 937-945.

5. Scanlan KA, Propeck PA, Lee FT Jr. Invasive Procedures in the Female Pelvis: Value of Transabdominal, Endovaginal, and Endorectal US Guidance. Radiographics 2001; 21: 491-497.

6. Weitmann HD, Pötter R, Waldhaus $C$ et al. Treatment of endometrial carcinoma with 3D image-based high dose rate brachytherapy using modified Heyman packing: clinical experience and dose volume histogram analysis. Int J Radiat Biol Phys 2005; 62: 468-478.

7. Person S, Hellström AC, Lundell M. HDR dose planning for intraoperative endometrial carcinoma with modified Heyman packing technique. Radiother Oncol 2005; 75 Suppl 1: S47.

8. Gerbaulet A, Pötter R, Mazeron JJ et al (eds.). GEC-ESTRO Handbook of Brachytherapy, Chapter 15.

9. Pálvölgyi J. To what extent can digital images obtained with a non-isocentric $\mathrm{C}$-arm be used for brachytherapy treatment planning in gynaecology. Radiother Oncol 2006; 67: 107-112.

10. Pálvölgyi J. Dose Distribution of Modified Heyman Packing. Physica Medica 2006; 22: 127-130. 